\title{
Zeno breaking, the Contact effect and sensitive behaviour in piecewise-linear systems $\dagger$
}

\author{
R. EDWARDS \\ Department of Mathematics, University of Victoria, PO Box 1700 STN CSC, Victoria, BC V8W 2Y2, Canada \\ email:edwards@uvic.ca
}

(Received 12 October 2017; revised 28 February 2018; accepted 28 February 2018; first published online

21 March 2018)

\begin{abstract}
Non-smooth approximations of steep sigmoidal switching networks, such as those used as qualitative models of gene regulation, lead to analytic and computational challenges that arise as a result of the discontinuities in the vector fields. In order to highlight the need for care in dealing with such systems, several particular phenomena are presented here through illustrative examples, including 'Zeno breaking', or computing beyond the finite time convergence of an infinite sequence of threshold transitions; the 'Contact' effect, in which in the discontinuous limit, trajectories can pass through a 'saddle point' without stopping, though these solutions are not unique and other solutions stop for arbitrary time intervals; and sensitive behaviour that arises from exotic dynamics within switching regions.
\end{abstract}

Key words: Discontinuous equations, singular perturbations, differential inclusions, oscillations, dynamical systems in biology (34A36; 34D15; 34A60; 34C10; 37N25)

\section{Introduction}

Non-smooth approximation to steep sigmoidal switching networks has proven to be a fruitful approach to analysis of their behaviour, for example, in the study of dynamics of gene regulation. However, the introduction of discontinuities also leads to some analytic challenges, and in situations where the true system is believed to be smooth, it is especially important to be sure that the behaviour predicted by the non-smooth analysis is close to that of nearby smooth systems, and to interpret results accordingly. Here, particular examples are used to highlight several challenges of this type, in which care needs to be taken in conducting or interpreting the non-smooth analysis. Some of the examples were inspired by analysis of gene-regulatory networks, but we are more concerned here with demonstrating the technical issues, in order to encourage rigorous analysis.

Autoregulation in piecewise-linear models of gene networks leads to sliding motion in threshold hyperplanes or their intersections, and trajectories in which two (or more) variables spiral in to threshold intersections in finite time. In the latter case of fast damped oscillation, an infinite number of threshold transitions occur during the finite time convergence to the intersection, as in Zeno's 'paradox'. After convergence, other

$\dagger$ This work was partially supported by a Discovery Grant from the Natural Sciences and Engineering Research Council (NSERC) of Canada. 
variables continue to evolve. This itself poses a computational challenge, since software systems designed to compute solution trajectories must be capable of computing past the Zeno convergence point. Ames [2] refers to such a point where simulators tend to get stuck as a "breaking (Zeno) point," though we could also use the term 'Zeno breaking' to refer to the ability of a good simulator to break out of the infinite loop and continue (reminiscent of 'breaking the sound barrier'). In gene networks with equal decay rates, we can compute explicitly the convergence time. We demonstrate by means of an example in Section 3. Qualitative models of gene networks are characterized by multilinear combinations of non-linearities, which are sigmoid in general, but step functions in the limiting piecewise-linear case. In Section 3.3, we consider both asymptotic and fast damped oscillation in an example system that is not multilinear, to demonstrate how long-term behaviour can depend sensitively on whether or not the threshold intersection is reached. Autoregulation in gene networks is actually accomplished by a sequence of steps, and introducing intermediate linear variables leads to more general piecewise-linear systems with other kinds of non-uniqueness, such as what we call here the 'Contact' effect, in which it becomes possible in the non-smooth limit of a saddle point to sail through it without stopping. Such an example is presented in Section 4. Exotic forms of behaviour through threshold regions, such as periodic or strange attractors in the boundary layer equations, can hide sensitive behaviour in nearby steep sigmoidal systems. In Section 5, we demonstrate this by means of another pair of examples.

\section{Continuous-time switching networks}

The motivating application for most of the examples that follow is that of gene-regulatory networks, in which piecewise-linear qualitative models have played a significant role for analysis of dynamical behaviours. However, the technical issues that are of concern in the current work relate to a much broader class of equations. All of the examples we deal with here will be piecewise-linear systems, with step function non-linearities, or smooth (but steep) sigmoidal perturbations of these. The class of systems can be expressed as follows:

$$
\dot{x}_{i}=F_{i}\left(Z_{1}\left(x_{1}\right), Z_{2}\left(x_{2}\right), \ldots, Z_{n}\left(x_{n}\right)\right)+G_{i}\left(x_{1}, x_{2}, \ldots, x_{n}\right), \quad i=1, \ldots, n,
$$

with linear functions $G_{i}$. In the context of Glass networks, which are often used to model gene regulation qualitatively, each $x_{i}$ is the concentration of the protein product of gene $i$, each $G_{i}=-\gamma_{i} x_{i}$, expressing degradation of the protein, each production term, $F_{i}$, is multilinear in $Z_{1}, \ldots, Z_{n}$, and each $Z_{i}\left(x_{i}\right)$ is a Hill function

$$
Z_{i}\left(x_{i}\right)=H\left(x_{i}, \theta_{i}, q\right)=\frac{x_{i}^{\frac{1}{q}}}{\theta_{i}^{\frac{1}{q}}+x_{i}^{\frac{1}{q}}}, \quad q \in(0,1] .
$$

In the limit as $q \rightarrow 0$,

$$
\lim _{q \rightarrow 0} H\left(x_{i}, \theta_{i}, q\right)=\left\{\begin{array}{lll}
0 & \text { if } & x_{i}<\theta_{i} \\
1 & \text { if } & x_{i}>\theta_{i}
\end{array}\right.
$$


and this becomes a non-smooth system where each $Z_{i}=0$ or 1 , except at thresholds. The Glass network structure is then,

$$
\dot{x}_{i}=F_{i}\left(Z_{1}\left(x_{1}\right), Z_{2}\left(x_{2}\right), \ldots, Z_{n}\left(x_{n}\right)\right)-\gamma_{i} x_{i}, \quad i=1, \ldots, n,
$$

with multilinear $F_{i}$.

In Section 3.3, we will present an example in which the functions $F_{i}$ are not multilinear in the $Z_{j}$, though $G_{i}=-\gamma_{i} x_{i}$ still. In Section 4 , we will use an example from a class of equations that extends Glass networks by including an additional linear variable for each regulatory equation (an mRNA concentration), so that half the equations have a multilinear $F_{i}$ with the standard degradation term, and the other half have $F_{i} \equiv 0$, but $G_{i}=\kappa_{i} x_{i-1}-\gamma_{i} x_{i}$ (though we will rename half the variables $y_{i}$ and reorder indices accordingly).

It is also possible for each gene product to have multiple thresholds (for example, a different threshold for the regulation of different genes). Then, we have $Z_{i j}=H\left(x_{i}, \theta_{i j}, q\right)$ for the regulation of the $j$ th gene by the $i$ th. We will not need to exploit this generalization to illustrate the behaviours we are interested in here.

The two main methods for dealing with the discontinuities in the vector fields are Filippov methods $[10,11]$, in which the equations are considered as differential inclusions, and potentially, set-valued solutions are possible and singular perturbation methods [14, 21], in which the non-smooth system is considered as a limit of smooth systems - in our case, the step functions are limits of underlying sigmoids. Much has been written on these methods, and we do not reproduce a full discussion here, but use them as needed.

However, it is important to point out that there are multiple definitions of Filippov solutions. At a point on one or more discontinuity surfaces (a codimension- $k$ switching domain is one in which $k$ variables are at their threshold, i.e., on their switching hyperplane), the usual approach defines the vector field as an element of the convex hull of the vectors at that point considered as part of the vector field in one of the smooth (regular) regions adjacent to the point. Thus, a point in a codimension- $k$ switching domain has $2^{k}$ adjacent regular regions. These have been called Filippov solutions in the general sense $[17,19]$. It has been argued in [17,19] that a more appropriate definition is one that allows all values across the discontinuous jump in each variable separately. In our context, this corresponds to allowing each $Z_{i}$ to take all values in the interval $[0,1]$. These have been called Filippov solutions in the narrow sense [17,19]. If the differential equations (in our case, the $F_{i}$ functions) are multilinear in the $Z_{i}$, then this definition corresponds to a multilinear interpolation of the vectors on each side of each discontinuity surface at a point. Others (e.g., [7,16]) have argued in a more general context for using a multilinear interpolation of these vectors, rather than the convex hull, but this approach only corresponds to the concept of Filippov solutions in the narrow sense if the differential equations are multilinear in the $Z_{i}$. There has been a profusion of names for these alternative definitions of vector field and solution, including Filippov solution in the narrow sense [17,19], Utkin's vector field or solution [7], the Aizermann-Pyatnitskii vector field [1], the bilinear interpolation vector field [6] or the convex canopy [16]. See also [12] for discussion of many of these papers. 
Table 1. Production terms of a general two-dimensional Glass network

\begin{tabular}{cc|cc}
\hline \hline$Z_{1}$ & $Z_{2}$ & $F_{1} / \gamma_{1}$ & $F_{2} / \gamma_{2}$ \\
\hline 0 & 0 & $a_{3}$ & $b_{3}$ \\
0 & 1 & $a_{2}$ & $b_{2}$ \\
1 & 0 & $a_{4}$ & $b_{4}$ \\
1 & 1 & $a_{1}$ & $b_{1}$ \\
\hline \hline
\end{tabular}

It is also important to recognize that the classical singular perturbation theory, based on Tikhonov's theorem, relies on asymptotic convergence of fast variables to a stable equilibrium, in order to continue the evolution of the slow variables in a way that ensures that behaviour of nearby smooth systems is still well approximated (at least for arbitrary finite time intervals) [21]. However, in [17,18], it was shown how an extension of this method due to Artstein and collaborators [3-5] allows continuation of solutions even when the attractor in the fast variables is not a fixed point.

\section{Zeno breaking}

\subsection{Oscillations: sustained, damped or fast damped}

The fact that finite-time convergence of damped oscillators can occur in Glass networks was first observed in [8]. The simplest setting is a two-gene system:

$$
\begin{aligned}
& \dot{y}_{1}=F_{1}\left(Z_{1}, Z_{2}\right)-\gamma_{1} y_{1}, \\
& \dot{y}_{2}=F_{2}\left(Z_{1}, Z_{2}\right)-\gamma_{2} y_{2},
\end{aligned}
$$

where we have translated thresholds to 0 via $y_{i}=x_{i}-\theta_{i}$, and $F_{1}$ and $F_{2}$ can in general be specified as in Table 1. To keep things simple, let us assume that the decay rates are equal, $\gamma_{1}=\gamma_{2}$.

For the structure in Figure 1, starting on the wall $\left\{y_{1}>0, y_{2}=0\right\}$, the map for the cycle through the four quadrants is

$$
M\left(y_{1}\right)=\frac{A y_{1}}{1+\phi y_{1}},
$$

where

$$
A=\frac{b_{1} a_{2} b_{3} a_{4}}{a_{1} b_{2} a_{3} b_{4}}, \quad \phi=-\frac{1}{a_{1}}+\frac{b_{1}}{a_{1} b_{2}}-\frac{b_{1} a_{2}}{a_{1} b_{2} a_{3}}+\frac{b_{1} a_{2} b_{3}}{a_{1} b_{2} a_{3} b_{4}} .
$$

For details of the calculations of these maps, see [8] or [9].

Oscillations are sustained (periodic orbit) if $A>1$ (see [20]). Oscillations are damped if $A \leqslant 1$. The time to convergence is finite if $A<1$ and infinite if $A=1$ (see [8]). If there is no autoregulation, then $A=1$.

\subsection{Computing past the Zeno point}

The Zeno phenomenon refers to finite-time convergence of an infinite sequence of transitions. Computer systems for analysis of piecewise-linear systems must deal with the 


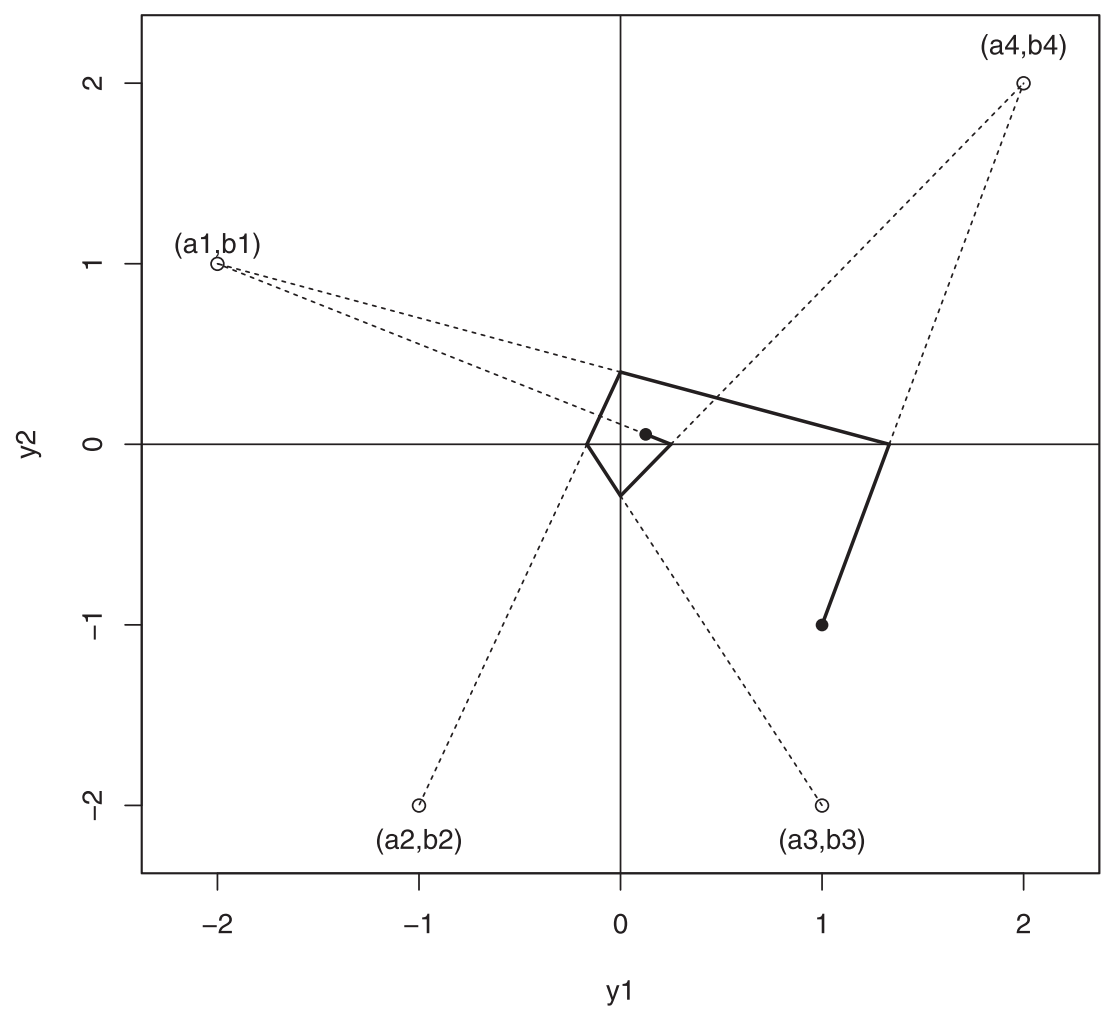

Figure 1. Damped oscillation in a two gene network.

problem of computing past the Zeno point (see, for example, [15]). A simulator that simply computes trajectories from transition to transition will 'break' at the 'Zeno (breaking) point' (for use of this term, see [2]). For Glass networks with equal decay rates, $\dot{y}=F(Z)-\gamma y\left(y \in \mathbb{R}^{n}\right)$, we can compute the Zeno point (time) directly, from an initial point $[8,9]$.

In the two-variable case, iteration of the cycle map ( $m$ times) yields

$$
M^{m}\left(y_{1}^{(0)}\right)=\frac{A^{m} y_{1}^{(0)}}{1+\phi^{(m, 0)} y_{1}^{(0)}},
$$

where the denominator is the exponential of the time taken, and

$$
\phi^{(m, 0)}=\sum_{k=0}^{m-1} A^{k} \phi=\frac{1-A^{m}}{1-A} \phi .
$$

Then, if $A<1$,

$$
\lim _{m \rightarrow \infty}\left(1+\phi^{(m, 0)} y_{1}^{(0)}\right)=1+\frac{\phi y_{1}^{(0)}}{1-A},
$$


so the time to convergence is

$$
t=\log \left(1+\frac{\phi y_{1}^{(0)}}{1-A}\right)
$$

If decay rates are not equal, explicit calculation of the convergence time is not so easy, but Ironi and coworkers implicitly deal with this in [14] and [15].

\section{Example 1}

$$
\begin{aligned}
& \dot{x}_{1}=2\left(1-Z_{2}\right)-x_{1}, \\
& \dot{x}_{2}=2.5 Z_{1}-0.5 Z_{1} Z_{2}-x_{2}, \\
& \dot{x}_{3}=1-0.1 x_{3},
\end{aligned}
$$

with $\theta_{1}=\theta_{2}=1$.

Here, $x_{1}$ and $x_{2}$ are independent of $x_{3}$ and have a phase plane like Figure 1. In terms of the shifted $y_{i}$ variables, $a_{1}=a_{2}=-1, a_{3}=a_{4}=1, b_{1}=1, b_{2}=b_{3}=-1$, $b_{4}=\frac{3}{2}$, and $A=\frac{2}{3}, \phi=\frac{11}{3}$, so the time to convergence to the threshold intersection is $T=\log \left(1+11 y_{1}^{(0)}\right)$. Recall that $y_{1}^{(0)}=x_{1}^{(0)}-1$, so $y_{1}^{(0)} \in(0, \infty)$.

Now $x_{3}$ continues to evolve, and there is sliding motion along the threshold intersection after $T=\log \left(1+11 y_{1}^{(0)}\right)$, asmpytotically converging to the singular stationary point at $\left(x_{1}, x_{2}, x_{3}\right)=(1,1,10)$. This can be established rigorously, by singular perturbation analysis, in terms of the boundary layer equations in $Z_{1}$ and $Z_{2}$.

\subsection{The Zeno phenomenon and sliding - a sensitive case}

To illustrate the potential sensitivity of a piecewise-linear system to the dynamics in the boundary layer system, we construct an example that is not multilinear like the Glass network equations.

\section{Example 2}

$$
\begin{aligned}
& \dot{x}_{1}=2-(5 / 3) Z_{2}-\alpha Z_{1} Z_{2}-x_{1}, \\
& \dot{x}_{2}=2 Z_{1}-x_{2}, \\
& \dot{x}_{3}=\left(2 Z_{1}-1\right)^{2}+\left(2 Z_{2}-1\right)^{2}-x_{3},
\end{aligned}
$$

with $\theta_{1}=\theta_{2}=1$, and $\alpha \in\left[-\frac{1}{3}, \frac{1}{3}\right]$. Figure 2 shows the behaviour for $\alpha=\frac{1}{3}, 0$ and $-\frac{1}{3}$.

\subsubsection{Singular perturbation analysis}

Note that if $Z_{1}, Z_{2} \in\{0,1\}$, then

$$
F_{3}\left(Z_{1}, Z_{2}\right)=\left(2 Z_{1}-1\right)^{2}+\left(2 Z_{2}-1\right)^{2}=2
$$

so $\dot{x}_{3}=2-x_{3}$ and $x_{3} \rightarrow 2$. This holds in all the regular regions around the threshold intersection $\left(x_{1}, x_{2}\right)=(1,1)$. Only when $Z_{1}$ or $Z_{2} \in(0,1)$ is $F_{3}\left(Z_{1}, Z_{2}\right)<2$. 

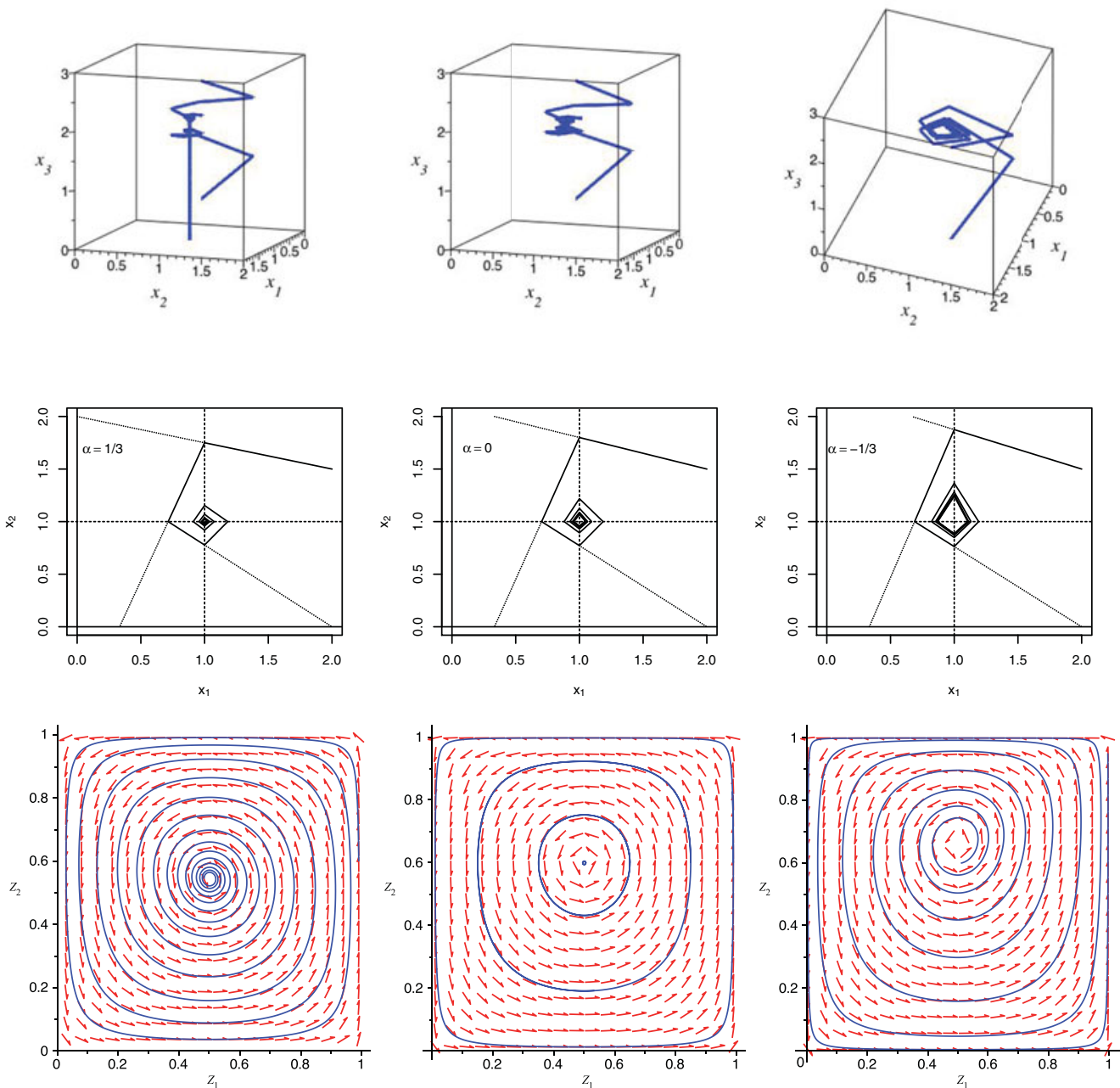

FIGURE 2. Behaviour of Example 2. The top panels show sample trajectories in the phase space for a small value of $q$; the middle row of panels show the flow in the $x_{1}, x_{2}$ projection of phase space in the limit $q \rightarrow 0$; the bottom panels show the behaviour of the fast variables in the $Z$-square (blow-up of the $x_{1}, x_{2}$ threshold intersection). In the left panels, $\alpha=\frac{1}{3}$ and $x_{3} \rightarrow \frac{1}{121}$ (in the limit $q \rightarrow 0$ ); in the middle column of panels, $\alpha=0$ and $x_{3} \rightarrow \frac{1}{25}$ or 2 , depending on $q$; in the right panels, $\alpha=-\frac{1}{3}$ and $x_{3} \rightarrow 2$ (in the limit $q \rightarrow 0$ ).

The equations for the fast dynamics in the switching intersection, $x_{1}=1, x_{2}=1$, can be obtained by expressing the first two equations of (3.1) in terms of the $Z$ variables using equation (2.2) to get

$$
\begin{aligned}
& Z_{1}^{\prime}=Z_{1}\left(1-Z_{1}\right)\left(1-(5 / 3) Z_{2}-\alpha Z_{1} Z_{2}\right), \\
& Z_{2}^{\prime}=Z_{2}\left(1-Z_{2}\right)\left(2 Z_{1}-1\right)
\end{aligned}
$$

where the derivatives are with respect to fast time, $\tau=\frac{t}{q}$ (see [21] for details of computing these boundary layer equations). The domain for these fast variables $\left([0,1]^{n}\right.$ in the 
switching intersection for $n$ variables) is called by Plahte and Kjøglum the $Z$-cube [21], but in two dimensions we can call it the $Z$-square. The fixed point in the switching intersection (found by setting the $Z_{1}^{\prime}$ and $Z_{2}^{\prime}$ to 0 ) is at $Z_{1}^{*}=\frac{1}{2}, Z_{2}^{*}=\frac{6}{10+3 \alpha}$. So, for example, at $\alpha=-\frac{1}{3}, 0$ and $\frac{1}{3}$, we have $Z_{2}^{*}=\frac{2}{3}, \frac{3}{5}$ and $\frac{6}{11}$, respectively. The Jacobian matrix is

$$
J\left(Z_{1}^{*}, Z_{2}^{*}\right)=\left[\begin{array}{cc}
-\frac{3 \alpha}{2(10+3 \alpha)} & -\frac{10+3 \alpha}{24} \\
\frac{12(4+3 \alpha)}{(10+3 \alpha)^{2}} & 0
\end{array}\right],
$$

so $\operatorname{det}(J)=\frac{4+3 \alpha}{2(10+3 \alpha)}>0$ for all $\alpha \in\left[-\frac{1}{3}, \frac{1}{3}\right]$ (the allowed range), and $\operatorname{tr}(J)<0$ if and only if $\alpha>0$ (within the allowed range). Also, $4 \operatorname{det}(J)>(\operatorname{tr}(J))^{2}$ for $\alpha \in\left[-\frac{1}{3}, \frac{1}{3}\right]$, so the fixed point is an asymptotically stable focus for $\alpha \in\left(0, \frac{1}{3}\right]$, and an unstable focus for $\alpha \in\left[-\frac{1}{3}, 0\right)$.

In the case $\alpha \in\left(0, \frac{1}{3}\right]$, solutions in the large phase space reach the threshold intersection in finite time, as can be calculated by the method above. $A=\frac{2}{3+2 \alpha}$ and $\phi=\frac{10}{2+3 \alpha}$, so the convergence time is $T=\log \left(1+\frac{\phi y_{1}^{(0)}}{1-A}\right)=\log \left(1+\frac{10}{3 \alpha} y_{1}^{(0)}\right)$, for a trajectory that begins on the $y_{1}>0, y_{2}=0$ wall (again $y_{i}=x_{i}-\theta_{i}$ ). At the Zeno point at $t=T$, trajectories of the fast variables enter the interior of the $Z$-square, and converge to the stable spiral point inside, at $\left(Z_{1}^{*}, Z_{2}^{*}\right)=\left(\frac{1}{2}, \frac{6}{10+3 \alpha}\right)$. This occurs instantaneously in terms of the normal time, $t$ (in the limit $q \rightarrow 0$ ). Then, the slow evolution of the $x_{3}$ equation is taken with $Z_{1}$ and $Z_{2}$ at these values, giving

$$
\dot{x}_{3}=\frac{(2-3 \alpha)^{2}}{(10+3 \alpha)^{2}}-x_{3},
$$

so that $x_{3} \rightarrow \frac{(2-3 \alpha)^{2}}{(10+3 \alpha)^{2}}$. See left panels of Figure 2 .

In the case $\alpha \in\left[-\frac{1}{3}, 0\right)$, the $Z$-square has no stable fixed point, solutions spiral out to the boundary, and the heteroclinic orbit around the boundary of the $Z$-square corresponds to solutions that cycle through the four regular domains in the large phase space. Indeed, the analysis of Section 3.1 show that there exists a periodic orbit, which can be shown to be stable by the methods of [8]. See right panels in Figure 2.

In the case $\alpha=0$, the Jacobian has pure imaginary eigenvalues, so a more delicate treatment is required. Nevertheless, we show below that the $Z$-square has a neutrally stable fixed point and nested periodic orbits, so again solutions cannot enter the interior of the $Z$-square from outside. This corresponds to the case in which solutions spiral into the threshold intersection, but in infinite time. See center column panels in Figure 2.

Let $\alpha=0$, and let $Y_{1}=Z_{1}-\frac{1}{2}$ and $Y_{2}=Z_{2}-\frac{1}{2}$, so that the fixed point $\left(Z_{1}^{*}, Z_{2}^{*}\right)=\left(\frac{1}{2}, \frac{3}{5}\right)$ becomes $\left(Y_{1}^{*}, Y_{2}^{*}\right)=\left(0, \frac{1}{10}\right)$, and the fast equations become

$$
\begin{aligned}
& Y_{1}^{\prime}=\left(\frac{1}{4}-Y_{1}^{2}\right)\left(\frac{1}{6}-\frac{5}{3} Y_{2}\right), \\
& Y_{2}^{\prime}=\left(\frac{1}{4}-Y_{2}^{2}\right)\left(2 Y_{1}\right) .
\end{aligned}
$$

Then,

$$
\frac{d Y_{2}}{d Y_{1}}=\frac{\left(\frac{1}{4}-Y_{2}^{2}\right)\left(2 Y_{1}\right)}{\left(\frac{1}{4}-Y_{1}^{2}\right)\left(\frac{1}{6}-\frac{5}{3} Y_{2}\right)}
$$


and

$$
\int \frac{\left(\frac{1}{6}-\frac{5}{3} Y_{2}\right)}{\left(\frac{1}{4}-Y_{2}^{2}\right)} d Y_{2}=\int \frac{2 Y_{1}}{\left(\frac{1}{4}-Y_{1}^{2}\right)} d Y_{1}
$$

which gives

$$
\frac{1}{3} \tanh ^{-1}\left(2 Y_{2}\right)+\frac{5}{6} \log \left(1-4 Y_{2}^{2}\right)=-\log \left(1-4 Y_{1}^{2}\right)+C
$$

or

$$
\log \left(1+2 Y_{1}\right)+\log \left(1-2 Y_{1}\right)+\log \left(1+2 Y_{2}\right)+\frac{2}{3} \log \left(1-2 Y_{2}\right)=C .
$$

A Taylor expansion about $\left(Y_{1}^{*}, Y_{2}^{*}\right)=\left(0, \frac{1}{10}\right)$ gives

$$
C \approx \log (1.2)+\frac{2}{3} \log (0.8)-4 Y_{1}^{2}-\frac{125}{36}\left(Y_{2}-\frac{1}{10}\right)^{2},
$$

and the Morse Lemma says that isoclines are closed curves (see, for example, [22]). Thus, orbits are closed curves around the fixed point, which is neutrally stable.

The global behaviour of the system in the large phase space for the case $\alpha=0$ is also sensitive. In the non-smooth system (i.e., in the limit $q \rightarrow 0$ ), the threshold intersection is never reached. Nevertheless, for any small $q>0$, the switching region can be defined to be of non-zero width, and solutions can enter it in finite time. This corresponds to the higher order singular perturbation analysis of Ironi and colleagues [14], which shows that under small perturbations of $q$ from 0 , the fixed point in the $Z$-square becomes stable. This can also be shown directly, by linearizing the sigmoidal system when $q>0$. The smooth version of the system (3.1) with $\alpha=0$ has $x_{1}$ nullcline $Z_{2}=\frac{3}{5}\left(2-x_{1}\right)$ and $x_{2}$ nullcline $Z_{1}=\frac{1}{2} x_{2}$, where $Z_{1}$ and $Z_{2}$ are smooth and monotonic functions of $q$, given by equation (2.2). The $x_{1}$ nullcline is decreasing (either considered as a function of $x_{1}$ or of $x_{2}$ ) and the $x_{2}$ nullcline is increasing. Given their ranges, it is clear that they have a unique intersection, $\left(x_{1}^{*}, x_{2}^{*}\right)$ which must approach $\left(\theta_{1}, \theta_{2}\right)=(1,1)$ as $q \rightarrow 0$. Then at an equilibrium, we must have $x_{3}=\left(2 Z_{1}-1\right)^{2}+\left(2 Z_{2}-1\right)^{2}=\left(x_{2}-1\right)^{2}+\left(\frac{7}{5}-\frac{6}{5} x_{1}\right)^{2}$ which approaches $\frac{1}{25}$ as $q \rightarrow 0$. This corresponds to the asymptotic value of $x_{3}$ in the finite-time convergence case.

\subsubsection{Filippov analysis}

Treating the $q=0$ system as a differential inclusion, there is a choice of definitions of Filippov solutions, as discussed in [17], Filippov solutions in the general sense or Filippov solutions in the narrow sense. The former use the convex hull of the focal points (or, equivalently, of the vector field), while the latter simply allows each $Z_{i}$ to take all values in [0,1]. It was shown in [17] that solutions in the narrow sense exist at least in codimension-1 or -2 switching regions. It is well known from Filippov that solutions in the general sense always exist [10].

The traditional Filippov solution uses the convex hull of focal points in the four regions around $\left(1,1, x_{3}\right): F_{11}=\left(\frac{1}{3}-\alpha, 2,2\right), F_{01}=\left(\frac{1}{3}, 0,2\right), F_{00}=(2,0,2), F_{10}=(2,2,2)$. The convex hull, $\Phi$, of the focal points intersects $\left(x_{1}, x_{2}\right)=(1,1)$ at $\phi=(1,1,2)$, regardless of the value of $\alpha$. So the Filippov solution in the general sense within the threshold intersection is a 


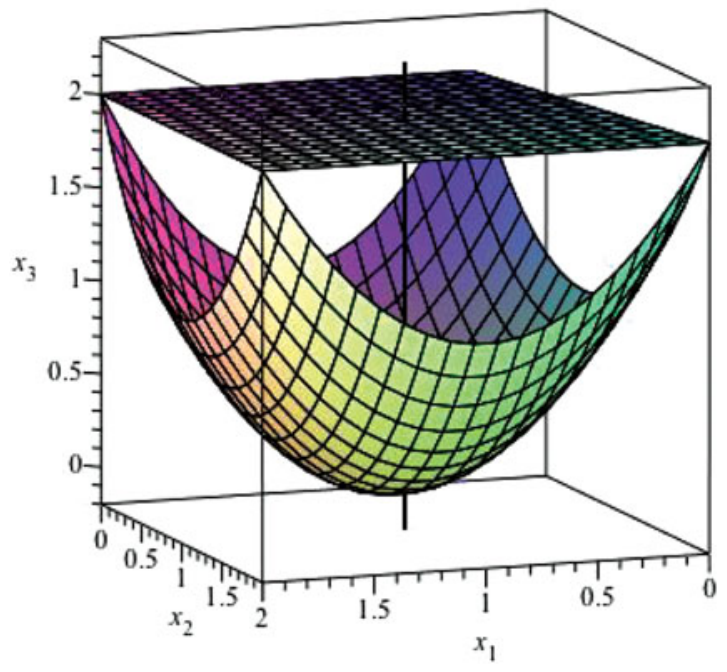

FIgURE 3. The flat surface is the set $\Phi$, the convex hull of the four focal points as used in finding Filippov solutions in the general sense. The parabolic surface, $\Phi_{1}$, is the corresponding set for finding Filippov solutions in the narrow sense. The vertical line is the intersection of thresholds $x_{1}=\theta_{1}=1, x_{2}=\theta_{2}=1$, whose intersection with $\Phi$ or $\Phi_{1}$ determines solutions.

trajectory flowing towards $(1,1,2)$ for any $\alpha \in\left[-\frac{1}{3}, \frac{1}{3}\right]$. A bilinear interpolation of the four focal points is in this case the same as the convex hull, so the same solution is obtained.

The Filippov solution in the narrow sense uses $Z_{i}=[0,1], i=1,2$. The set of potential focal points is a paraboloid

$$
\Phi_{1}=\left\{2-\frac{5}{3} Z_{2}-\alpha Z_{1} Z_{2}, \quad 2 Z_{1}, \quad\left(2 Z_{1}-1\right)^{2}+\left(2 Z_{2}-1\right)^{2}\right\},
$$

which intersects $\left(x_{1}, x_{2}\right)=(1,1)$ when $\left(Z_{1}, Z_{2}\right)=\left(\frac{1}{2}, \frac{6}{10+3 \alpha}\right)$ at

$$
\phi_{1}=\left(1,1,\left(2 Z_{1}-1\right)^{2}+\left(2 Z_{2}-1\right)^{2}\right)=\left(1,1, \frac{(2-3 \alpha)^{2}}{(10+3 \alpha)^{2}}\right),
$$

(see Figure 3). This agrees with the singular perturbation fixed point.

Technically, the Filippov method does not allow conclusions to be drawn about behaviour after the infinite sequence of transitions of the damped oscillation. However, from within the threshold intersection, it is only the narrow sense of solutions that corresponds to the singular perturbation solutions, at least when $\alpha \neq 0$.

The significance of Example 2 is that $x_{3}$ dropping to near zero may represent an important event for the system modelled (e.g., cell death). If $\alpha \in\left[-\frac{1}{3}, 0\right.$ ), then this event certainly does not happen, and all methods agree. If $\alpha \in\left(0, \frac{1}{3}\right]$, then the Filippov solution in the general sense and the bilinear interpolation approach say that this event does not happen, while the Filippov solution in the narrow sense and the singular perturbation solution say that it does. If $\alpha=0$, then the Filippov method in any sense says the event does not happen, but for nearby smooth systems, it does happen, and it is only the 
extended singular perturbation analysis by Ironi and colleagues [14] or a direct calculation on the smooth system that shows this.

\section{Grazing and the 'Contact' effect}

In this section, following [13], we model gene networks with two variables per gene, one for mRNA concentration $\left(x_{i}\right)$ and one for protein concentration $\left(y_{i}\right)$ :

$$
\begin{aligned}
& \dot{x}_{i}=F_{i}(Z)-\beta_{i} x_{i} \\
& \dot{y}_{i}=\kappa_{i} x_{i}-\gamma_{i} y_{i}
\end{aligned} \quad i=1, \ldots, n,
$$

where $Z=Z_{1}, \ldots, Z_{n}$, and each $Z_{i}=H\left(y_{i}, \theta_{i}, q\right)$, a function of $y_{i}$ instead of $x_{i}$.

The $i$ th production term, $F_{i}$, does not contain $x_{i}$. This means that no variable appears in its own equation. Every wall (threshold) is 'transparent' - trajectories cannot hit a threshold and stay there. Autoregulation of a gene now shows up as damped oscillations instead of hitting a wall and sliding. They may, however, spiral into an asymptotically stable fixed point on a threshold or at an intersection of thresholds. Thus, singular dynamics (sliding, the Zeno phenomenon) are largely avoided. However, it is now possible for trajectories to graze thresholds (intersect threshold hyperplanes tangentially).

'Boxes' or 'Domains' for this system are bounded by thresholds only in the $y_{i}$ variables, while the $x_{i}$ have no thresholds (other than being bounded above 0 ). In the limit $q \rightarrow 0$, in a given domain $Z$ is a constant vector, so $\alpha_{i}=F_{i}(Z)$ is constant.

Thus, in a given domain, the system is

$$
\begin{aligned}
& \dot{x}_{i}=\alpha_{i}-\beta_{i} x_{i} \\
& \dot{y}_{i}=\kappa_{i} x_{i}-\gamma_{i} y_{i}
\end{aligned} \quad i=1, \ldots, n,
$$

The focal point for the domain is given by

$$
\phi=\left(x^{*}, y^{*}\right)=\left(x_{1}^{*}, \ldots, x_{n}^{*}, y_{1}^{*}, \ldots, y_{n}^{*}\right) \text { where }\left(x_{i}^{*}, y_{i}^{*}\right)=\left(\frac{\alpha_{i}}{\beta_{i}}, \frac{\kappa_{i} \alpha_{i}}{\gamma_{i} \beta_{i}}\right) .
$$

Solution trajectories within and between domains can be computed.

Fixed points in regular domains are asymptotically stable, though some trajectories starting in a domain with a fixed point leave it. Moreover, trajectories are non-monotone in $y_{i}$ within a domain, so flow can cross walls in both directions. There are solutions that graze the wall from both sides at the same point, leading to non-uniqueness again in the limit $q \rightarrow 0$ (see Figure 4). These trajectories define upper or lower boundaries, $\Gamma_{u_{i}}^{(j)}$ or $\Gamma_{l_{i}}^{(j)}$, of invariant regions within each domain (see [13]).

A grazing point, perturbed from $q=0$, occurs in situations that can be expressed canonically as

$$
\begin{aligned}
& \dot{x}=b+a Z-\beta x, \\
& \dot{y}=\kappa x-\gamma y,
\end{aligned}
$$

where $a, b \geqslant 0$.

The following result comes from [13], though the statement of the proposition there had a typographical error in the condition on $\theta$, and a more complete proof is provided here. 


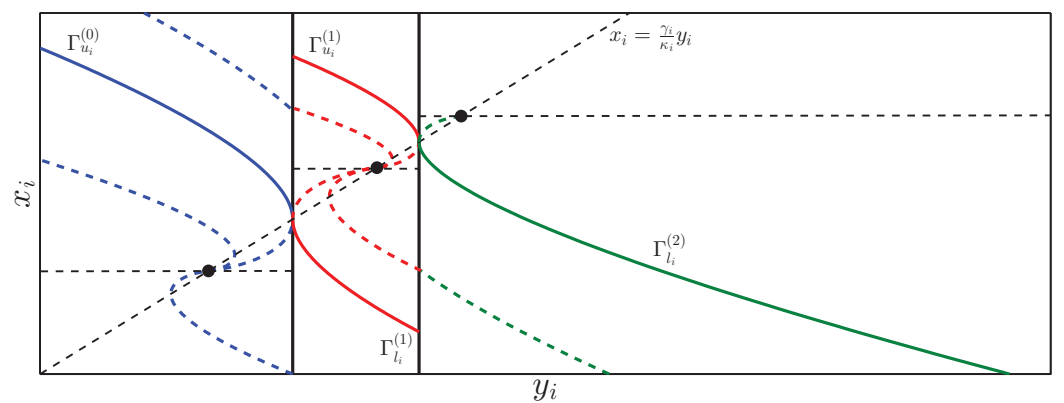

Figure 4. Dynamics of the two variables corresponding to a single gene, $y_{i}$ is protein concentration, $x_{i}$ is mRNA concentration. Two thresholds are depicted for the protein. The straight black dotted lines are nullclines. The grazing trajectories are denoted $\Gamma_{u_{i}}^{(j)}$ and $\Gamma_{l_{i}}^{(j)}$ in the domain indicated by $j=0,1,2$, where the subscripts $u_{i}$ and $l_{i}$ describe the fact that these trajectories define upper and lower boundaries of an invariant region in each domain.

Proposition 1 If $b<\frac{\beta \gamma}{\kappa} \theta<a+b$, then there is a fixed point for the pair $(x, y)$ in a neighbourhood of $\left(\frac{\gamma}{\kappa} \theta, \theta\right)$. Moreover, this point converges to $\left(\frac{\gamma}{\kappa} \theta, \theta\right)$ as $q \rightarrow 0$. If $q$ is sufficiently small, then this fixed point is a saddle point.

Proof of Proposition 1 The $x$ and $y$ nullclines can be written, respectively, as

$$
x=f_{1}(y)=\frac{1}{\beta}\left(b+a \frac{y^{\frac{1}{q}}}{y^{\frac{1}{q}}+\theta^{\frac{1}{q}}}\right) \quad \text { and } \quad x=f_{2}(y)=\frac{\gamma}{\kappa} y .
$$

Note that $f_{1}(y)$ is a shifted Hill function starting at $x=\frac{b}{\beta}$ when $y=0$ and increasing as $y$ increases, approaching $x=\frac{b+a}{\beta}$ as $y \rightarrow \infty$. The assumption of the proposition implies that $\theta \in\left(\frac{b \kappa}{\beta \gamma}, \frac{(b+a) \kappa}{\beta \gamma}\right)$. In this case, as $q \rightarrow 0$, the Hill function approaches a step function, with a jump at $y=\theta$, so that for $q$ sufficiently small, there exist three intersections of the nullclines. To see this, recall that for the Hill function, $\lim _{q \rightarrow 0} H(y, \theta, q)=0$ for any fixed $y<\theta$ and $\lim _{q \rightarrow 0} H(y, \theta, q)=1$ for any fixed $y>\theta$. Thus, $f_{1}(y)<f_{2}(y)$ for any fixed $y \in\left(\frac{b \kappa}{\beta \gamma}, \theta\right)$ for sufficiently small $q$, and $f_{1}(y) \rightarrow \frac{b}{\beta}$ as $q \rightarrow 0$. Similarly, $f_{1}(y)>f_{2}(y)$ for any fixed $y \in\left(\theta, \frac{(b+a) \kappa}{\beta \gamma}\right)$, for sufficiently small $q$, and $f_{1}(y) \rightarrow \frac{b+a}{\beta}$ as $q \rightarrow 0$. Clearly, $f_{1}(0)=\frac{b}{\beta}>0=f_{2}(0)$ for all $q$, and $f_{1}(y)<\frac{b+a}{\beta}<f_{2}(y)$ for $y>\frac{(b+a) \kappa}{\beta \gamma}$, so there are three points where $f_{1}(y)=f_{2}(y)$ for sufficiently small $q>0$. At the end-points, $f_{1}\left(\frac{b \kappa}{\beta \gamma}\right) \rightarrow \frac{b}{\beta}=f_{2}\left(\frac{b \kappa}{\beta \gamma}\right)$, and $f_{1}\left(\frac{(b+a) \kappa}{\beta \gamma}\right)=\frac{b+a}{\beta}=f_{2}\left(\frac{(b+a) \kappa}{\beta \gamma}\right)$ as $q \rightarrow 0$, which give the asymptotic values of the two outer fixed points. These are clearly not in the vicinity of the threshold.

For the middle fixed point, express the $x$ nullcline as a function of $y$ :

$$
y=\left(\frac{\beta x-b}{a+b-\beta x}\right)^{q} \theta .
$$

Now, as $q \rightarrow 0$, for any point $x$ in the open interval $\left(\frac{b}{\beta}, \frac{b+a}{\beta}\right)$, it is clear that $y \rightarrow \theta$, so the transition region of the Hill function provides the third intersection of the nullclines at a point that approaches $\left(\frac{\gamma}{\kappa} \theta, \theta\right)$ as $q \rightarrow 0$. 
The Jacobian of the system is

$$
J(x, y)=\left(\begin{array}{cc}
-\beta & \frac{a \theta^{\frac{1}{q}} y^{\frac{1}{q}-1}}{q\left(y^{\frac{1}{q}}+\theta^{\frac{1}{q}}\right)^{2}} \\
\kappa & -\gamma
\end{array}\right)
$$

which at the central fixed point, $(\bar{x}, \bar{y})$, has eigenvalues

$$
\lambda_{1,2}=-\frac{\beta+\gamma}{2} \pm \sqrt{\frac{(\beta+\gamma)^{2}}{4}+\frac{a \kappa \theta^{\frac{1}{q}} \bar{y}^{\frac{1}{q}-1}}{q\left(\bar{y}^{\frac{1}{q}}+\theta^{\frac{1}{q}}\right)^{2}}-\beta \gamma}
$$

which approach $\pm \infty$ as $q \rightarrow 0$, since

$$
\lim _{q \rightarrow 0} \frac{a \kappa \theta^{\frac{1}{q}} \bar{y}^{\frac{1}{q}-1}}{q\left(\bar{y}^{\frac{1}{q}}+\theta^{\frac{1}{q}}\right)^{2}}=\lim _{q \rightarrow 0} \frac{a \kappa \theta^{\frac{1}{q}} \theta^{\frac{1}{q}}\left(\frac{\beta \bar{x}-b}{a+b-\beta \bar{x}}\right)}{q\left(\theta^{\frac{1}{q}}\left(\frac{\beta \bar{x}-b}{a+b-\beta \bar{x}}\right)+\theta^{\frac{1}{q}}\right)^{2} \bar{y}}=\lim _{q \rightarrow 0} \frac{a \kappa\left(\frac{\beta \bar{x}-b}{a+b-\beta \bar{x}}\right)}{q\left(\left(\frac{\beta \bar{x}-b}{a+b-\beta \bar{x}}\right)+1\right)^{2} \bar{y}}
$$

which $\rightarrow \infty$, because $\bar{x}$ is strictly in the interior of the interval $\left(\frac{b}{\beta}, \frac{b+a}{\beta}\right)$.

Thus, the grazing point is a saddle point with the rate of approach to the fixed point on the stable manifold approaching $\infty$ as $q \rightarrow 0$, and the rate of approach to the fixed point in reverse time on the unstable manifold similarly approaches $\infty$ as $q \rightarrow 0$. For any $q>0$, the solution takes infinite time to reach the saddle on the stable manifold, or leave the saddle on the unstable manifold. At $q=0$, the solution can sail right through the 'fixed point' as if it were not there: $e^{-\lambda t}$ reaches 0 for finite $t$ in the limit $\lambda \rightarrow \infty$. In fact, two solutions pass through the same 'fixed point', but the non-uniqueness is more profound than this: the solution trajectory may stop at the 'fixed point' or follow either continuation. In fact, Webber and colleagues have shown that there is a 'temporal indeterminacy' in that the solution may stop at the grazing point for an arbitrary time interval before continuing [23].

The fact that the solution may sail right through the saddle point in the $q \rightarrow 0$ limit, however, means that the infinite time interval spent in the vicinity of the 'fixed point' for a solution on the stable/unstable manifold can disappear from view at $q=0$. It is reminiscent of the interstellar journey taken by Ellie (played by Jodie Foster) in the film 'Contact', which motivated the term, 'Contact effect'. In the film, the capsule in which Ellie sits is dropped into an energy field created by a spherical machine built according to instructions from extra-terrestrials. From the outside, the capsule appears to drop straight through the sphere, but from Ellie's point of view, at the centre point of the sphere, she enters a wormhole and goes on a long trip through space before returning to fall the rest of the way through the sphere. Somehow, all the action at the centre point is squeezed into zero units of real time, as if an infinite eigenvalue were at work! 


\section{Masking of sensitivity in the discontinuous limit}

The possibility of complex behaviour in the fast variables in switching domains was introduced in [17], in which it was shown by example that, within the multilinear structure of gene network models, fast variables could converge to periodic orbits or strange attractors, rather than fixed points, necessitating an extension, due to Artstein and collaborators [3-5], of the classical singular perturbation theory. The periodic example there was a three-dimensional system based on the Rössler system, but with parameters in the periodic, rather than chaotic regime. In that example, all three variables reach their thresholds in finite time, and remain there, but the fast variables in the codimension-3 switching domain converge to a periodic orbit, called a 'quasi-Rössler' attractor. In [18], this example was extended to a four-dimensional system, in which a fourth variable continued to evolve slowly, while the other three executed their microscopic periodic orbit within their triple threshold intersection. The subsequent motion depended sensitively on the location on the periodic orbit of the first three variables at the moment the fourth hit its threshold. There were two possible continuations, each converging to a different fixed point. For small $q$, the basins of attraction of the two fixed points are densely interwoven, implying a kind of sensitivity to initial conditions, despite the fact that trajectories starting close to each other remain close for a long time, during the slow evolution of the fourth variable, only to diverge after it switches. The sensitivity increases as $q$ decreases. In the limit $q \rightarrow 0$, the interweaving of the two basins of attraction becomes infinitely refined and the eventual fate of a trajectory cannot be determined. We are left with non-uniqueness.

\subsection{Quasi-Rössler controlled by another loop}

Here, we demonstrate another phenomenon that can arise from such a structure, in which the system displays complexity of behaviour as a result of a kind of sensitivity to initial conditions for small $q>0$, which is lost (or masked) in the limit $q \rightarrow 0$. We show this by extending the original quasi-Rössler system by two additional variables, as follows.

\section{Example 3}

$$
\begin{aligned}
& \dot{x}_{1}=5\left(Z_{1}-\frac{1}{2}\right) Z_{4}+\frac{1}{2}-Z_{2}-\frac{1}{4} Z_{3}+\gamma_{1} \theta_{1}-\gamma_{1} x_{1}, \\
& \dot{x}_{2}=Z_{1}-\frac{1}{2}+a\left(Z_{2}-\frac{1}{2}\right)+\gamma_{2} \theta_{2}-\gamma_{2} x_{2}, \\
& \dot{x}_{3}=b+Z_{3}\left(18 Z_{1}-10-c\right)+\gamma_{3} \theta_{3}-\gamma_{3} x_{3}, \\
& \dot{x}_{4}=\frac{1}{2}-Z_{5}+\frac{1}{4} Z_{4} Z_{5}+\gamma_{4} \theta_{4}-\gamma_{4} x_{4}, \\
& \dot{x}_{5}=Z_{4}-\frac{1}{2}+\gamma_{5} \theta_{5}-\gamma_{5} x_{5},
\end{aligned}
$$

with the parameter values $a=0.2, b=0.4, c=5.7, \gamma_{1}=\gamma_{2}=\gamma_{3}=10, \gamma_{4}=\gamma_{5}=0.1$, and thresholds for the $Z_{i}$ given by $\theta_{1}=\theta_{2}=0.3, \theta_{3}=1.6, \theta_{4}=\theta_{5}=5$.

Note that $x_{4}$ and $x_{5}$ are independent of the other variables, and simply approach a periodic orbit. Their low decay rates make them evolve more slowly than $x_{1}, x_{2}$ and $x_{3}$. The quasi-Rössler example in the first three variables is recovered when $Z_{4}=0$, i.e., when $x_{4}<\theta_{4}$. 
Let $y_{i}=x_{i}-\theta_{i}, i=1, \ldots, 5$ so that each threshold, $\theta_{i}$, is moved to the origin, and consider the transformed system

$$
\begin{aligned}
& \dot{y}_{1}=5\left(Z_{1}-\frac{1}{2}\right) Z_{4}+\frac{1}{2}-Z_{2}-\frac{1}{4} Z_{3}-\gamma_{1} y_{1}, \\
& \dot{y}_{2}=Z_{1}-\frac{1}{2}+a\left(Z_{2}-\frac{1}{2}\right)-\gamma_{2} y_{2}, \\
& \dot{y}_{3}=b+Z_{3}\left(18 Z_{1}-10-c\right)-\gamma_{3} y_{3}, \\
& \dot{y}_{4}=\frac{1}{2}-Z_{5}+\frac{1}{4} Z_{4} Z_{5}-\gamma_{4} y_{4}, \\
& \dot{y}_{5}=Z_{4}-\frac{1}{2}-\gamma_{5} y_{5},
\end{aligned}
$$

where $Z_{i}=H\left(y_{i}+\theta_{i}, \theta_{i}, q\right), i=1, \ldots, 5$.

First, we describe the behaviour of this system in the limit $q \rightarrow 0$. It can be shown in a straightforward manner by the method of [8] that $y_{4}$ and $y_{5}$ in equation (5.2), considered as an independent system, converge to a periodic orbit around the origin. This corresponds, of course, to an oscillation about the threshold intersection of $x_{4}$ and $x_{5}$ in equation (5.1). Now, $Z_{4}=0$ while $y_{4}<0$, and during intervals of time when this holds, $y_{1}, y_{2}$ and $y_{3}$ converge to their threshold intersection in finite time, as shown in [17]. When $y_{4}$ reaches 0 , we enter a codimension-4 switching region, and with our choice of parameter values, $a, b$ and $c$, the trajectory can exit the $\left(Z_{1}, Z_{2}, Z_{3}, Z_{4}\right)$ box in two places, $(0,0, b /(10+c), 1)$ or $(1,1,1,1)$, as can be shown by an analysis of the boundary layer equations in fast time [18]. The choice of exit points is not determined in the $q \rightarrow 0$ limit. In either case, we exit with $Z_{4}=1$, so $y_{4}$ goes positive, and $y_{4}$ and $y_{5}$ continue around their cycle.

From the first exit point, $\left(Z_{1}, Z_{2}, Z_{3}, Z_{4}\right)=(0,0, b /(10+c), 1)=\left(0,0, \frac{4}{157}, 1\right)$, we enter the domain where $y_{1}$ and $y_{2}$ are negative, $y_{3}$ is still 0 and $y_{4}$ is positive. Now, according to equations (5.2), $y_{1}$ approaches $-\frac{1}{5}-\frac{1}{1570}$, which is slightly less than -0.2 , while $y_{2} \rightarrow-0.06$. The corresponding values in the original system (5.1) are $x_{1} \approx 0.1$ and $x_{2}=0.24$. Eventually, $y_{4}$ switches off again, and $y_{1}, y_{2}$ and $y_{3}$ again converge to 0 in finite time (in this case, $y_{3}$ remained at 0 throughout the excursion in $y_{1}$ and $y_{2}$ ). Then, $y_{4}$ evolves slowly towards its next switching and the process repeats.

From the second exit point, $\left(Z_{1}, Z_{2}, Z_{3}, Z_{4}\right)=(1,1,1,1)$, we enter the region in which $y_{1}, y_{2}, y_{3}$ and $y_{4}$ are all positive. Now, $y_{1} \rightarrow 0.175, y_{2} \rightarrow 0.06$ and $y_{3} \rightarrow 0.27$. This focal point corresponds to $x_{1}=0.475, x_{2}=0.36, x_{3}=1.87$ in the original system. Again, when $y_{4}$ switches off again, we repeat the process.

Figure 5 shows the $x_{1}, x_{2}$ projection of a trajectory of (5.1) with parameter values as listed above and $q=0.003$. The initial point was taken as $\left(x_{1}, x_{2}, x_{3}, x_{4}, x_{5}\right)=$ $(0.5,0.4,0.6,4.9,5.5)$. The threshold intersection is at $(0.3,0.3)$ in this plot, and the small oscillations near this point are clear, corresponding to the Rössler cycling in the codimension3 switching domain. Then repeated excursions occur on loop $A$, towards a point near $(0.1,0.24)$ and back, and on loop $B$, towards the point $(0.475,0.36)$ and back.

The two excursions in $y_{1}$ and $y_{2}$ (and $y_{3}$ in the second case), emanating from the two exit points, we denote loop $A$ and loop $B$. In the $q \rightarrow 0$ limit, the trajectories from the two exit points both emanate from $\left(y_{1}, y_{2}, y_{3}, y_{4}\right)=(0,0,0,0)$ exactly and there are only two possible subsequent trajectories, corresponding to loops $A$ and $B$. Of course, the choice of trajectory from the codimension- 4 switching domain is not determined, and thus, in principle, following a circuit of loop $A$, we could next take either $A$ or $B$ and similarly after 


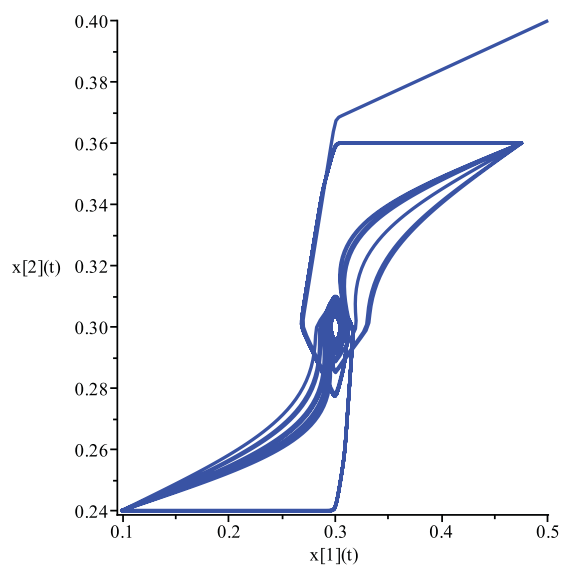

(a)

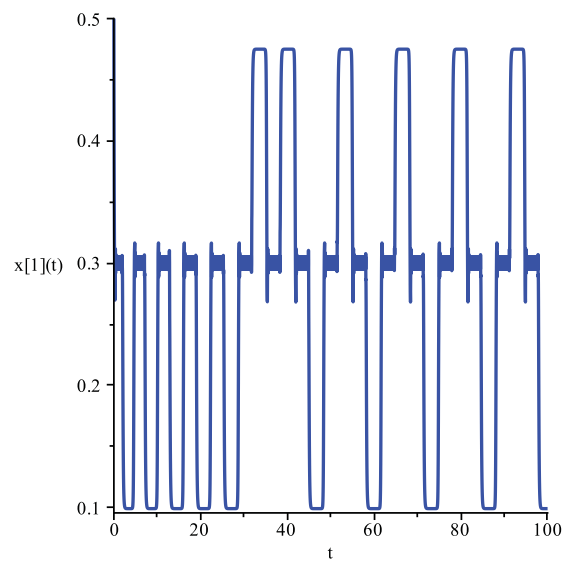

(b)

FiguRE 5. Evolution of a trajectory of (5.1) with parameter values as given in the text. (a) The projection of the phase space onto $\left(x_{1}, x_{2}\right)$, showing the two loops $(A$ to the lower left, $B$ to the upper right, and the small oscillations near the threshold intersection at $(0.3,0.3)$. (b) Time plot of $x_{1}$, from which one can read the sequence of loop traversals. Downward excursions correspond to loop $A$; upward excursions correspond to loop $B$.

$B$. When $q>0$, however, the exact trajectory depends, sensitively, on the previous history. A slight change in the point where $y_{1}, y_{2}$ and $y_{3}$ reach their codimension-3 switching domain (now of small but finite width), leads to a slight change in where the trajectory exits from the codimension-4 switching domain, and thus a slight change in the exact trajectory along loop $A$ or loop $B$ and on the choice of the next cycle. The sensitive dependence generated by the oscillation in the codimension-3 switching domain leads to sensitivity in the determination of the next loop, $A$ or $B$. Whether there are stable periodic orbits here that involve some sequence of loops $A$ and $B$, or whether we have chaos is not clear. For the example trajectory in Figure 5, the itinerary appears to be $A^{5} B^{2}(A B)^{n}$, falling eventually into a periodic cycle through $A B$.

In the $q \rightarrow 0$ limit, we certainly do not have chaos in the usual sense, only nonuniqueness, since there is only a single trajectory following each of loop $A$ and loop $B$. Indeed, in that case, if we believe that input to the codimension-3 switching domain at exactly the same point should lead to exactly the same subsequent trajectory, then the loop following loop $A$ or $B$ would be fixed. Then there would be only two possible itineraries, one beginning with $A$, and one with $B$. The non-uniqueness, however, implies that either choice can always be made, so any itinerary is possible, but with no way to determine which will be chosen. This is very different from the well-defined sensitive dependence of the system with $q>0$.

\subsection{Periodic fast motion in a codimension-2 switching region}

Although we used a codimension-3 switching region above to contain the periodic orbit in the three fast variables (the quasi-Rössler attractor), it should be noted that periodic solutions can occur even in a codimension- 2 switching region and even with linear $F_{i}$ (i.e., 
they need not even be multilinear). This may appear surprising, since a linear system in itself cannot have limit cycles, and an unstable focus implies that solutions away from the focus spiral out to infinity. However, in the $Z$-square expansion of a codimension-2 switching region, the differential equations for the fast variables are multiplied by the factors $Z_{i}\left(1-Z_{i}\right) / \theta_{i}$ (this is a consequence of using Hill functions as sigmoids). Thus, if the $F_{i}$ are linear, the boundary layer equations are cubic, and are clearly bounded by the boundary of the $Z$-square itself. The combination of an unstable focus and the boundedness imposed by the fast equations makes limit cycle solutions possible.

An example of the latter type is given by Del Buono and colleagues in equation (3.5) of [7].

$$
\begin{aligned}
& \dot{x}_{1}=\left(-2 Z_{1}-Z_{2}+\frac{33}{10}\right)-x_{1}, \\
& \dot{x}_{2}=\left(8 Z_{1}+\mu\left(Z_{2}-\frac{1}{2}\right)-\frac{67}{10}\right)-\frac{1}{2} x_{2}, \\
& \dot{x}_{3}=\left(1-Z_{1}-Z_{2}+2 Z_{1} Z_{2}\right)-\frac{1}{3} x_{3},
\end{aligned}
$$

where $\theta_{1}=\theta_{2}=1$, and $\mu$ is a parameter. The regime in which the periodic solution in the codimension- 2 switching domain $\left(Z_{1}, Z_{2}\right)$ occurs is $\frac{18}{25}<\mu<\frac{8}{5}$. The boundary layer equations are

$$
\begin{aligned}
& Z_{1}^{\prime}=Z_{1}\left(1-Z_{1}\right)\left(-2 Z_{1}-Z_{2}+\frac{23}{10}\right) \\
& Z_{2}^{\prime}=Z_{2}\left(1-Z_{2}\right)\left(8 Z_{1}+\mu\left(Z_{2}-\frac{1}{2}\right)-\frac{36}{5} .\right.
\end{aligned}
$$

In the given parameter region, the Poincaré-Bendixson theorem can be used to prove existence of a periodic solution, since the rectangle $Z_{1} \in[0.65,1], Z_{2} \in[0,1]$ is invariant and contains only an unstable focus in the interior at $(0.9,0.5)$ (and saddle points at $(1,0),(1,1)$ and $(0.65,1)$, which can be shown not be in $\omega$-limit sets for any initial point in the interior of the rectangle. It would be easy to extend this system to display the same type of sensitive behaviour as the quasi-Rössler system of [18] or that of Example 3 above.

\section{Discussion}

A fruitful discussion has developed between those who seek to model smooth systems using discontinuous limits as an analytic tool, and those who seek to study non-smooth systems via regularization across the discontinuities. In the former case, one has prior information about the nature of the discontinuity (or one could say the appropriate regularization) from the form of the underlying smooth system, and if one employs nonsmooth techniques, it is important to know how the resulting solutions compare to those of 'nearby' smooth systems (i.e., small smooth perturbations of the non-smooth system).

On another front, attempts are being made to develop software tools to study the behaviour of non-smooth models of gene networks, or steep sigmoidal models that use perturbation from the non-smooth case. This involves not only computing sliding solutions in switching domains, but dealing with the Zeno effect. Filippov methods have difficulty with the latter from an analytic point of view. Some progress on the Zeno breaking problem has been made $[8,14]$, but we show here by example that in the borderline case of asymptotic (infinite-time) convergence, care must be taken to ensure that the behaviour of an underlying smooth system is properly captured. Filippov methods do not give the solution that matches that of nearby smooth systems, which a careful (higher order) 
singular perturbation analysis can, or direct analysis of the smooth system if it is simple enough to handle (like the one used here).

In a very simple example, we demonstrate the Contact effect, a kind of non-uniqueness in the discontinuous limit in which multiple solutions can sail through a fixed point, or stop there for an arbitrary length of time. Such a point arises (in our example) as the limit of a saddle point in nearby smooth systems. The surprise is that no matter how small the perturbation from the non-smooth limit (our $q$ parameter), it takes an infinite amount of time to reach the saddle point along its stable manifold, whereas in the limit it takes a finite time. This parallels the case of the Zeno effect, in which infinite-time convergence to a stable focus (in the variables that are spiralling) in the smooth systems leads to finite-time convergence in the non-smooth limit.

The possibility of exotic behaviour (periodic or strange attractors) in codimension-3 switching regions of piecewise-linear gene network models has necessitated use of more sophisticated singular perturbation tools that integrate over the attractor in the fast variables in order to track the slow ones. It has also raised the possibility of a kind of sensitive dependence on initial conditions in steep sigmoidal systems that becomes more sensitive as one approaches the non-smooth limit, but in the non-smooth limit the details of this sensitive behaviour are lost, and only non-uniqueness remains. It has also become clear from an example in [7] that such exotic behaviour (limit cycles in fast variables) can occur even in codimension-2 switching regions and even when the fast system is linear (not even multilinear).

This set of examples is presented here in order to provide test cases for analytic and computational methods, and to encourage rigorous analysis of such systems.

\section{References}

[1] Acary, V., De Jong, H. \& Brogliato, B. (2014) Numerical simulation of piecewise-linear models of gene regulatory networks using complementarity systems. Physica D 269, 103119.

[2] Ames, A. D., Zheng, H., Gregg, R. D. \& SAstry, S. (2006) Is there life after Zeno? Taking executions past the breaking (Zeno) point. In: Proceedings of the 2006 American Control Conference, 14-16 June 2006, Minneapolis, Minnesota: IEEE, 2652-2657.

[3] ARtstein, Z. (2002) On singularly perturbed ordinary differential equations with measurevalued limits. Math. Bohemica 127(2), 139-152.

[4] Artstein, Z., Linshiz, J. \& Titi, E. S. (2007) Young measure approach to computing slowly advancing fast oscillations. Multiscale Model. Simul. 6(4), 1085-1097.

[5] Artstein, Z. \& Vigodner, A. (1996) Singularly perturbed ordinary differential equations with dynamic limits. Proc. R. Soc. Edinburgh: Sect. A Math. 126(3), 541-569.

[6] Dieci, L. \& Difonzo, F. (2014) A comparison of Filippov sliding vector fields in codimension 2. J. Comput. Appl. Math. 262, 161-179.

[7] Del Buono, N., Elia, C. \& Lopez, L. (2014) On the equivalence between the sigmoidal approach and Utkin's approach for piecewise-linear models of gene regulatory networks. SIAM J. Appl. Dyn. Syst. 13(3), 1270-1292.

[8] Edwards, R. (2000) Analysis of continuous-time switching networks. Physica D 146, 165-199.

[9] Edwards, R., Hill, A. \& Jacquier, M. (2014) Analysis of transient damped oscillations in gene regulatory networks. In: Proceedings of the MTNS Conference, July 2014, Groningen, the Netherlands. 
[10] Filippov, A. F. (1988) Differential Equations with Discontinuous Righthand Sides, Kluwer, Dordrecht.

[11] GouzÉ, J.-L. \& SARI, T. (2002) A class of piecewise linear differential equations arising in biological models. Dyn. Syst. 17(4), 299-316.

[12] Guglielmi, N. \& Hairer, E. (2015) Classification of hidden dynamics in discontinuous dynamical systems. SIAM J. Appl. Dyn. Syst. 14(3), 1454-1477.

[13] Hudson, D. \& Edwards, R. (2016) Dynamics of transcription-translation networks. Physica D 331, 102-113.

[14] Ironi, L., Panzeri, L., Plahte, E. \& Simoncini, V. (2011) Dynamics of actively regulated gene networks. Physica D 240(8), 779-794.

[15] Ironi, L. \& Tran, D. X. (2016) Nonlinear and temporal multiscale dynamics of gene regulatory networks: A qualitative simulator. Math. Comput. Simul. 125, 15-37.

[16] JEFFrey, M. R. (2014) Dynamics at a switching intersection: Hierarchy, isonomy, and multiple sliding. SIAM J. Appl. Dyn. Syst. 13(3), 1082-1105.

[17] Machina, A., Edwards, R. \& van den Driessche, P. (2013) Singular dynamics in gene network models. SIAM J. Appl. Dyn. Syst. 12(1), 95-125.

[18] Machina, A., Edwards, R. \& van den Driessche, P. (2013) Sensitive dependence on initial conditions in gene networks. Chaos 23(2), 025101.

[19] Machina, A. \& Ponosov, A. (2011) Filippov solutions in the analysis of piecewise linear models describing gene regulatory networks. Nonlinear Anal. 74(3), 882-900.

[20] Mestl, T., Plahte, E. \& Oмholt, S. W. (1995) Periodic solutions in systems of piecewise-linear differential equations. Dyn. Stab. Syst. 10(2), 179-193.

[21] Plahte, E. \& KJøglum, S. (2005) Analysis and generic properties of gene regulatory networks with graded response functions. Physica D 201(1-2), 150-176.

[22] Verhulst, F. (1996) Nonlinear Differential Equations and Dynamical Systems, 2nd ed., Springer, Berlin.

[23] Webber, S., Glendinning, P. \& Jeffrey, M. R. (2018) Pausing in piecewise-smooth dynamic systems, in preparation. 